\title{
A simulation study on the performance of the ALPAQUITA experiment
}

\section{S. Kato ${ }^{a}$ on behalf of the ALPACA Collaboration}

(a complete list of authors can be found at the end of the proceedings)

${ }^{a}$ Institute for Cosmic Ray Research, University of Tokyo, Kashiwa, Chiba 277-8582, Japan

E-mail: katosei@icrr.u-tokyo.ac.jp

The ALPACA experiment is a new air shower experiment mainly aiming to explore the southern sky in the VHE gamma-ray regime beyond $100 \mathrm{TeV}$. As the prototype experiment, ALPAQUITA will start in late 2021. It consists of a surface air shower array $\left(18,450 \mathrm{~m}^{2}\right)$ and an underground muon detector array $\left(900 \mathrm{~m}^{2}\right)$. In this study, the performance of ALPAQUITA including the sensitivity to gamma-ray point sources is investigated using a Monte Carlo simulation to quantitatively evaluate the possibility of detection of gamma-ray sources in the prototype phase. Corsika 7.6400 and Geant4 v10.04.p02 are used to simulate air shower development in the atmosphere and detector response, respectively. The output data are then processed and analyzed in the same way as the experiment. As a result, the study finds that the air shower array has an energy resolution of $\pm 21 \%$ and the angular resolution of $\simeq 0.2^{\circ}$ for gamma rays with an energy of $100 \mathrm{TeV}$. The detection area of the air shower array for gamma rays reaches $\simeq 12,600 \mathrm{~m}^{2}$ above $\simeq 30 \mathrm{TeV}$. The muon detector rejects $\simeq 99.9 \%$ of background cosmic rays and maintains $\simeq 80 \%$ of signal gamma rays. This high discrimination power will enable the detection of five southern known gamma-ray sources beyond $30 \mathrm{TeV}$ and the extension of the energy spectrum of one out of the five, HESS J1702-420A, up to $\simeq 300 \mathrm{TeV}$ during one calendar year observation. This study concludes that ALPAQUITA will provide data enough to discuss a hot topic of VHE gamma-ray astronomy before passing the baton to ALPACA.

$3^{\text {th }}$ International Cosmic Ray Conference (ICRC 2021)

July 12 th $-23 r d, 2021$

Online - Berlin, Germany 


\section{Introduction}

The origin of the knee region of the cosmic-ray energy spectrum has been one of the biggest mysteries of cosmic-ray physics for over half a century [1]. Observation of gamma rays beyond $100 \mathrm{TeV}$ is an effective way to specify PeVatrons, which are thought to accelerate cosmic rays up to the knee energy in the Galaxy. VHE gamma-ray astronomy beyond $100 \mathrm{TeV}$ has been opened by Tibet AS $\gamma$ [2] [3], HAWC [4] [5] [6] [7] and LHAASO [8] [9]. These experiments are exploring the northern sky in the highest energy gamma-ray regime and have discovered several candidates of PeVatron. Tibet AS $\gamma$ also discovered that PeVatrons certainly exist or existed in the past in the Galaxy from the observation of galactic diffuse gamma rays up to $1 \mathrm{PeV}$ [10].

However, the southern sky should be a more attractive area to observe. This is because the Galactic Center and the ambient high star density region can be seen in the southern sky. The Galactic plane survey performed by H.E.S.S. revealed about 100 gamma-ray sources up to several tens of TeV [11]. The discoveries include several PeVatron candidates such as Galactic Center [12], HESS J1702-420A and HESS J1702-420B [13]. Also interestingly, for a large portion of the gamma-ray sources, no plausible counterparts are detected in other wavelength ranges [11]. Under these circumstances, the top priority should be put on observing as many sources as possible and extending the energy spectra beyond $100 \mathrm{TeV}$, leading to further activation of multi-wavelength study. Therefore, experiments sensitive to gamma rays beyond $100 \mathrm{TeV}$ in the southern hemisphere are eagerly awaited.

Now the ALPACA experiment is proceeding aiming to explore VHE gamma-ray astronomy beyond $100 \mathrm{TeV}$ in the southern hemisphere, and the prototype experiment ALPAQUITA will start its operation in 2021. This paper introduces the simulation study on the performance of ALPAQUITA including the sensitivity to southern gamma-ray sources and discusses the detectability of the sources in its short period of observation before starting ALPACA. In Section 2, the detector configuration of ALPAQUITA is briefly introduced. Following Section 3 and 4 describe the simulation settings and data analysis procedure, respectively. Results of the simulation analysis are presented in Section 5, and finally, Section 6 concludes the paper. For the current status of the ALPACA project and the simulation study on ALPACA, see the discussions by S. Sako [14] and Y. Yokoe [15], respectively.

\section{The ALPAQUITA experiment}

ALPAQUITA is an air shower array experiment, consisting of a surface air shower array (AS array) and a muon detector (MD) placed beneath the ground (see Figure 1). The AS array is composed of 97 scintillation detectors and has a geometrical area of 18,450 $\mathrm{m}^{2}$. Each scintillation detector records the energy loss and the detection timing of the secondary particles of extensive air showers to reconstruct energies and incoming directions of primary particles. MD, with a geometrical area of $900 \mathrm{~m}^{2}$, is centered at the AS array and composed of 16 smaller cell units. Each cell contains water and collects Cherenkov light mainly emitted by penetrating muons in air showers. MD enables us to discriminate shower events induced by signal gamma rays from those by background cosmic rays, leading to a great improvement of the experiment's sensitivity to gamma-ray sources. 


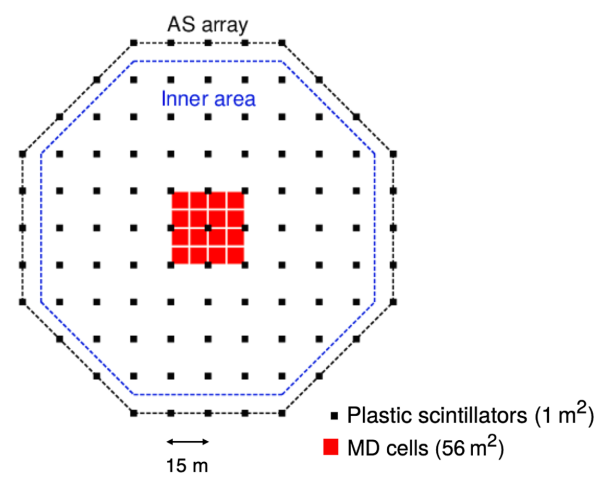

Figure 1: Detector configuration of ALPAQUITA. For the inner area surrounded by dashed blue lines, see Section 4.2.

\section{Monte Carlo simulation}

\subsection{Air shower generation}

Corsika7.6400 [16] is used for the generation of both gamma-ray and cosmic-ay induced air shower events. Primary particles are injected into the atmosphere along the path in the sky of RX J1713.73946 and shower development is simulated. For the chemical composition and the energy spectrum of cosmic rays, the model proposed by Shibata et al. (2010) [17] is applied. For gamma rays, a simple power-law spectrum with an index of 2 is assumed. However, the index is modified in the later analysis by appropriately weighting the events. Shower cores are distributed randomly over the circular region which has a radius of $300 \mathrm{~m}$ and is centered at the ALPAQUITA AS array. Table 1 summarizes the Corsika simulation settings in this study.

Table 1: Corsika simulation settings.

\begin{tabular}{ccc}
\hline Primary particles & Gamma rays & Cosmic rays \\
\hline Interaction model & EGS4 & FLUKA \& EPOS-LHC \\
\hline Energy range & \multirow{2}{*}{$300 \mathrm{GeV}<E<10 \mathrm{PeV}$} & $300 \mathrm{GeV}<E<10 \mathrm{PeV}$ \\
& $\& 10 \mathrm{TeV}<E<10 \mathrm{PeV}$ \\
\hline Total number of events & $3.7 \times 10^{7}$ & $1.1 \times 10^{8} \& 7.7 \times 10^{7}$ \\
\hline Spectrum & $\propto E^{-2}$ & M. Shibata et al. $(2010)[17]$ \\
\hline Path in the sky & RX J1713.7-3946 $\left(\theta_{\min }=23.4^{\circ}\right)$ \\
\hline Simulation area & Circular region with a 300 m radius from the AS array center \\
\hline
\end{tabular}

\subsection{Detector response}

The detector response to shower events is simulated with Geant4 v10.04.p02 [18]. For the AS array, energy loss process of shower particles in each scintillation detector is simulated. In this study, the single-particle peak of the scintillation detectors is defined as $9.4 \mathrm{MeV}$, and a trigger is issued when any four detectors record more than 0.5 particles within a $600 \mathrm{~ns}$ gate. Each detector records the total energy loss of shower particles and the average detection timing of the deposit. 
MD is located underground at the center of the AS array with a $2 \mathrm{~m}$ soil overburden. For the shower particles that reach MD, their Cherenkov light emission and the paths in the water layer are simulated. The outputs of all the PMTs are obtained as the number of photoelectrons, and the single muon peak is defined as 24 photoelectrons in this study.

\section{Data analysis}

\subsection{Reconstruction method}

Raw data obtained in the simulation is processed in the same way as the experiment and then used for the data analysis. In this process, the shower core position, incoming direction, and energy of a primary particle are reconstructed. The shower core position is estimated with the following weighted average of the positions of scintillation detectors with respect to recorded particle density $\rho_{i}$ :

$$
\left(\frac{\sum_{i} \rho_{i}^{1.5} x_{i}}{\sum_{i} \rho_{i}^{1.5}}, \frac{\sum_{i} \rho_{i}^{1.5} y_{i}}{\sum_{i} \rho_{i}^{1.5}}\right)
$$

where $x_{i}$ and $y_{i}$ are the coordinates of the $\mathrm{i}$-th scintillation detector.

For the estimation of the incoming direction, assuming that a shower front has a conical shape, the relative detection timing of the i-th scintillation detector $t_{i}$ is modified as

$$
t_{i}^{\prime}=t_{i}-b r_{i}
$$

where $b(\mathrm{~ns} / \mathrm{m})$ is the slope of the cone and $r$ is the distance between the shower axis and the $\mathrm{i}$-th detector. Then incoming direction $\boldsymbol{l}$ is calculated so that it minimizes the following quantity called "residual error" $\chi$ :

$$
\chi^{2}=\sum_{i} w_{i}\left(\boldsymbol{l} \cdot \boldsymbol{x}_{\boldsymbol{i}}-c\left(t_{0}-t_{i}^{\prime}\right)\right)^{2} \quad\left(w_{i}=\frac{\rho_{i}}{\sum_{j} \rho_{j}}, \quad \boldsymbol{x}_{\boldsymbol{i}}=\left(x_{i}, y_{i}, 0\right)\right)
$$

where $c$ is the light velocity and $t_{0}$ is the relative timing of the shower core reaching the AS array.

Energy reconstruction is performed with two different estimation methods depending on the number of scintillation detectors used for the direction reconstruction (Equation (3)). If the number of detectors is smaller than 30 , then the energy is estimated with $\sum \rho$, the total particle density detected with the AS array minus the maximum contribution, and the distance between the AS array center and the reconstructed shower core position. Otherwise, energy is estimated using the NKG function fitted to a reconstructed lateral shower distribution. In this study, particle density at the point $40 \mathrm{~m}$ distant from the shower axis (S40) is employed.

\subsection{Analysis conditions}

After reconstructing the events, several analysis conditions are imposed. The following five conditions are employed to evaluate the AS array performance:(1) any four scintillation detectors record more than 0.8 particle density, (2) the detector that records the largest particle density is inside the inner area shown in Figure 1, (3) the residual error in Equation (3) is smaller than $1 \mathrm{~m}$, (4) for the events whose energies are estimated with the S40 method, the reconstructed age parameter is 
smaller than 1.3, and (5) reconstructed zenith angle is smaller than $40^{\circ}$. For the conditions applied to the analysis using MD, see Section 5.2.

\section{Results}

\subsection{The AS array performance}

Energy resolution Figure 2 shows one of the energy distributions (left) obtained in this study and the resultant energy resolution of the ALPAQUITA AS array for gamma rays. In the right figure, the upper (lower) energy resolution is defined as a $1 \sigma$ standard deviation of the asymmetric Gaussian distribution fitted to the right (left) side with respect to the peak (refer to the left figure). With this definition, the energy resolution of $\pm 21 \%$ can be achieved in the $100 \mathrm{TeV}$ reconstructed energy range.
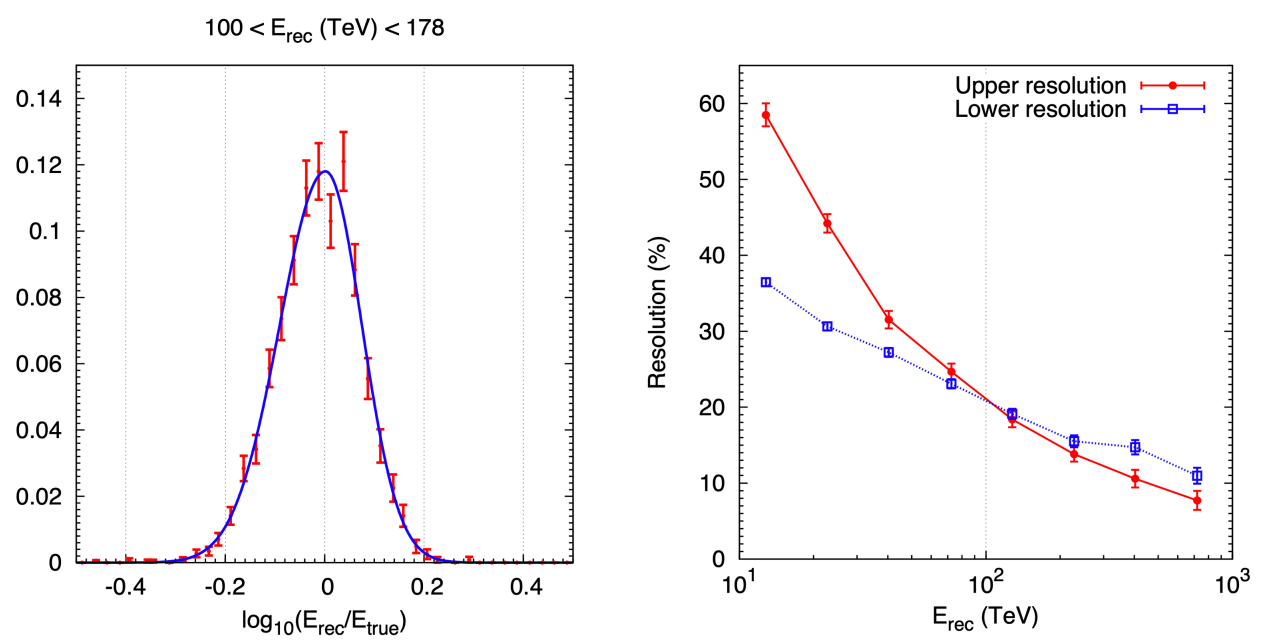

Figure 2: Left: distribution of gamma-ray events whose reconstructed energies are between $100 \mathrm{TeV}$ and $178 \mathrm{TeV}$. The blue curve shows the resultant asymmetric Gaussian fitted to the distribution. Right: energy resolution for gamma rays.

Angular resolution Figure 3 shows the angular resolution of the ALPAQUITA AS array for gamma rays. The resolution improves monotonically with increasing the energy range, and in the $100 \mathrm{TeV}$ reconstructed energy range, it reaches $0.21^{\circ}$ and $0.27^{\circ}$ as $50 \%$ and $68 \%$ containment radii, respectively.

Detection area The detection area of the ALPAQUITA AS array for gamma rays is shown in Figure 4 . The area asymptotes the geometrical size of the inner area $\left(12,600 \mathrm{~m}^{2}\right)$ because the analysis condition (2) (refer to Section 4.2) rejects the shower events that make the outermost detectors record the largest particle densities. In the reconstructed energy range from $\simeq 20 \mathrm{TeV}$ to $1 \mathrm{PeV}$, the detection area is stably constant. 


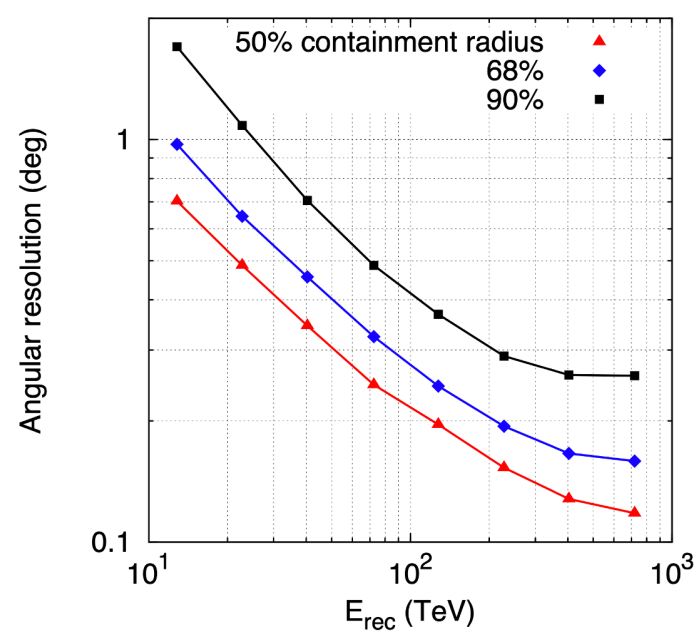

Figure 3: Angular resolution for gamma rays. Error radii inside which $50 \%, 68 \%$, and $90 \%$ of events are contained are shown.

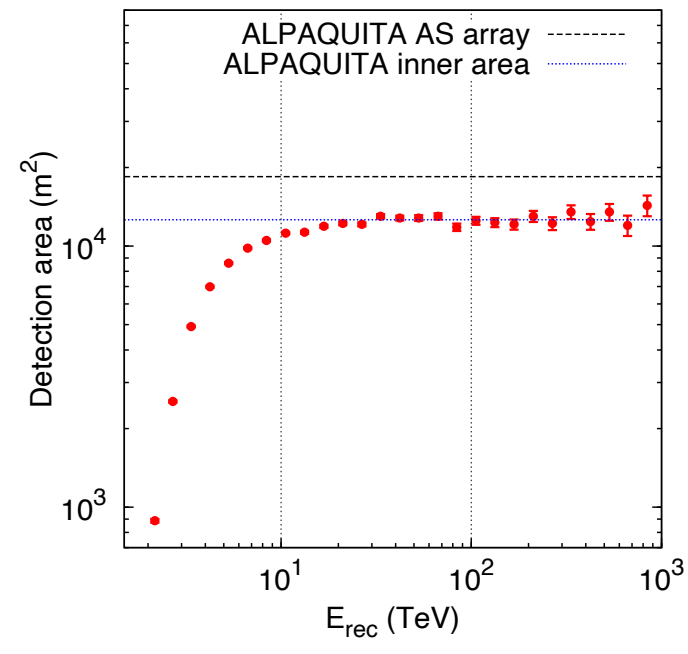

Figure 4: Detection area of the ALPAQUITA AS array for gamma rays. For the inner area, see Figure 1 .

\subsection{The MD performance}

Analysis conditions In the analysis using MD, the following analysis condition is further imposed on events in addition to those described in Section 4.2: incoming direction of an event must be within the window that is centered at a source position and have a radius of

$$
r= \begin{cases}1.5^{\circ} & \left(\Sigma \rho<15 \mathrm{~m}^{-2}\right), \\ \frac{5.8^{\circ}}{\sqrt{\Sigma \rho / \mathrm{m}^{-2}}} & \left(15 \mathrm{~m}^{-2}<\Sigma \rho<135 \mathrm{~m}^{-2}\right), \text { and } \\ 0.5^{\circ} & \left(135 \mathrm{~m}^{-2}<\Sigma \rho\right) .\end{cases}
$$

This condition improves the Q-factor of gamma-ray events and is optimized for ALPAQUITA.

Event selection criterion using MD Figure 5 shows the gamma-ray and background cosmic-ray events scattered in the $\left(\Sigma \rho, \Sigma N_{\mu}\right)$ plane, where $\Sigma N_{\mu}$ is defined as the total number of muons detected with MD. It is seen that cosmic-ray-induced showers give much larger muon signals to MD than gamma-ray-induced ones due to the hadronic interaction. The optimum event cut line using $\Sigma N_{\mu}$ is also drawn in the figure. Applying this cut can reject $\simeq 99.9 \%$ of background cosmic rays while maintaining $\simeq 80 \%$ of signal gamma rays.

Sensitivity to gamma-ray sources In Figure 6, the ALPAQUITA sensitivity curve to a gammaray point source (thick black line) is overlapped to gamma-ray energy spectra of several southern gamma-ray sources. It is seen that the ALPAQUITA has the capability of detecting five sources beyond several tens of TeV in its one calendar year observation and that one source, HESS J1702$420 \mathrm{~A}$, will be detected beyond $100 \mathrm{TeV}$ in the same observational duration. 


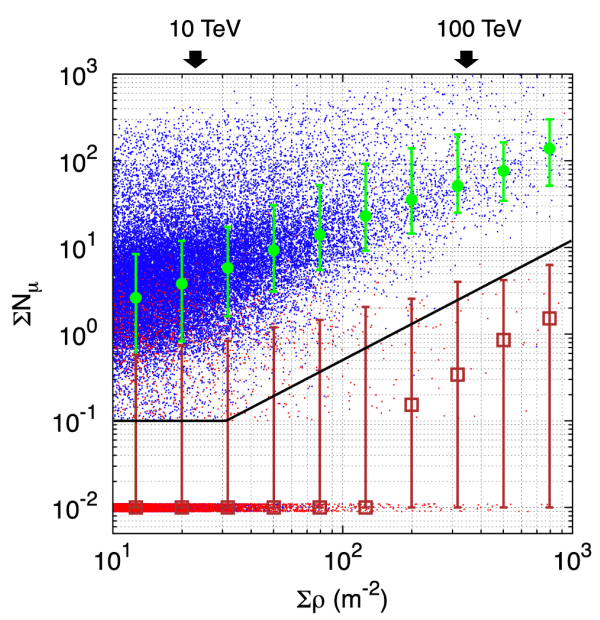

Figure 5: Gamma-ray (red) and background cosmic-ray (blue) events scattered in the $\left(\Sigma \rho, \Sigma N_{\mu}\right)$ plane. For both kinds of events, $\Sigma N_{\mu}$ values which contain $16 \%, 50 \%$, and $84 \%$ of events are shown with vertical bars. Events whose $\Sigma N_{\mu}$ is smaller than 0.1 are piled up at $\Sigma N_{\mu}=0.01$. Also shown is the optimum event cut line that maximizes the Q-factor of signal gamma rays.

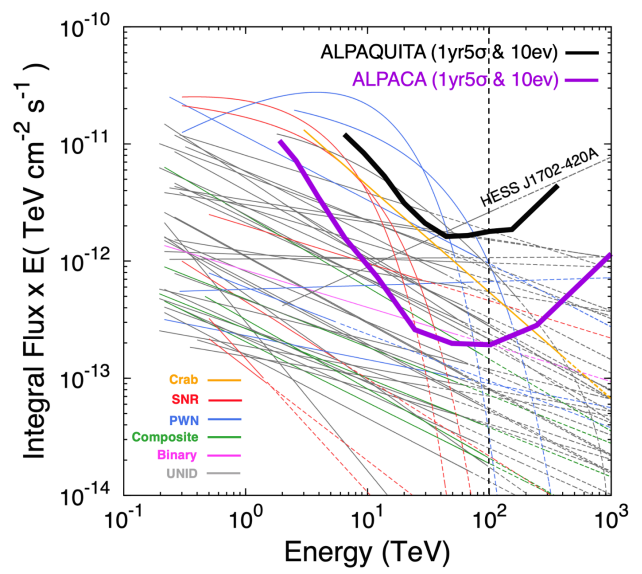

Figure 6: Sensitivity curve of ALPAQUITA to gamma-ray point source (thick black). For reference, the ALPACA sensitivity curve is also shown (thick purple). Thin curves show gammaray energy spectra of several gamma-ray sources [2] [5] [11] and solid and dashed lines indicate observed and extrapolated regions, respectively. Source types are distinguished with colors. For HESS J1702-420A, see the text.

HESS J1702-420A This peculiar source has been recently reported by H.E.S.S. experiment together with HESS J1702-420B, which is spatially overlapping. HESS J1702-420A is a point-like source and has a hard photon index of $\simeq 1.5$ extending up to $\simeq 100 \mathrm{TeV}$. ALPAQUITA will be able to plot flux points up to $\simeq 300 \mathrm{TeV}$ with sufficient accuracy if assuming no cutoff in the energy spectrum (refer to Figure 6), and contribute to disentangling the gamma-ray emission of this PeVatron candidate. Therefore, ALPAQUITA has an interesting scientific mission in its short observational period of instrumental validation.

\section{Conclusion}

The performance of ALPAQUITA including the sensitivity to a gamma-ray point source is investigated. The study shows that ALPAQUITA will enable us to discuss a hot topic of VHE gamma-ray astronomy and make its short period of observation fruitful. This activates the authentic start of the search for gamma-ray sources beyond $100 \mathrm{TeV}$ in the southern hemisphere before ALPACA starts its operation.

\section{Acknowledgement}

The ALPACA project is supported by the Japan Society for the Promotion of Science (JSPS) through Grants-in-Aid for Scientific Research (A) 19H00678, Scientific Research (B) 19H01922, and Scientific Research (S) 20H05640, the LeoAtrox supercomputer located at the facilities of 
the Centro de Análisis de Datos (CADS), CGSAIT, Universidad de Guadalajara, México, and by the joint research program of the Institute for Cosmic Ray Research (ICRR), The University of Tokyo. K. Kawata is supported by the Toray Science Foundation. E. de la Fuente thanks Coordinación General Académica y de Innovación (CGAI-UDG), cuerpo académico PRODEPUDG-CA-499, Carlos Iván Moreno, Cynthia Ruano, Rosario Cedano, and Diana Naylleli, for financial and administrative support during sabbatical year stay at the ICRR on 2021. I. ToledanoJuarez acknowledges support from CONACyT, México; grant 754851.

\section{References}

[1] G.V. Kulikov, G.B. Kristiansen, Zh. Eksp. Teor. Fis. 35, 635 (1958)

[2] M. Amenomori et al., Physical Review Letters 123, 051101 (2019)

[3] The Tibet AS $\gamma$ Collaboration, Nature Astronomy 5, 460 (2021)

[4] A. U. Abeysekara et al., The Astrophysical Journal 881, 134 (2019)

[5] A. U. Abeysekara et al., Physical Review Letters 124, 021102 (2020)

[6] A Albert et al., The Astrophysical Journal Letters 896, L29 (2020)

[7] A. Albert et al., Nature Astron 5, 465 (2021)

[8] F. Aharonian et al., Chinese Phys. C 45, 025002 (2021)

[9] Cao, Z., Aharonian, F.A., An, Q. et al., Nature 594, 33 (2021)

[10] M. Amenomori et al. (Tibet AS $\gamma$ Collaboration), Physcal Review Letters 126, 141101 (2021)

[11] HESS Collaboration, Astronomy \& Astrophysics 612, A1 (2018)

[12] HESS Collaboration, Nature 531, 476 (2016)

[13] H. Abdalla et al., arXiv:2106.06405v2

[14] T. Sako et al. (ALPACA Collaboration), Proc. of ICRC2021, PoS (ICRC2021) 777 (2021)

[15] Y. Yokoe et al. (ALPACA Collaboration), Proc. of ICRC2021, PoS (ICRC2021) 947 (2021)

[16] D. Heck, J. Knapp, J.N. Capdevielle, G. Schats, T. Thouw, Report FZKAp. 6019 (1998)

[17] M. Shibata, Y. Katayose, J. Huang, D. Chen, The Astrophysical Journal 716, 1076 (2010)

[18] S. Agostinelli, et al., Nucl. Instrum. Methods Phys. Res. A 506, 250 (2003) 


\section{Full Authors List: ALPACA Collaboration}

F. Aceves de la Cruz ${ }^{1}$, S. Asano ${ }^{2}$, C. A. H. Condori ${ }^{3}$, E. de la Fuente ${ }^{1,4}$, J. L. Garcia-Luna ${ }^{1}$, A. Gomi ${ }^{5}$, K. Hibino ${ }^{6}$, N. Hotta ${ }^{7}$, A. Jimenez-Meza ${ }^{8}$, Y. Katayose ${ }^{9}$, C. Kato ${ }^{2}$, S. Kato ${ }^{10}$, T. Kawashima ${ }^{10}$, K. Kawata ${ }^{10}$, T. Koi ${ }^{11}$, H. Kojima ${ }^{12}$, D. Kurashige ${ }^{5}$, J. Lozoya ${ }^{13}$, R. Mayta ${ }^{14,15}$, P. Miranda ${ }^{3}$, K. Munakata ${ }^{2}$, K. Nagaya ${ }^{5}$, Y. Nakamura ${ }^{10}$, Y. Nakazawa ${ }^{16}$, C. Nina ${ }^{3}$, M. Nishizawa ${ }^{17}$, S. Ogio ${ }^{14,15}$, M. Ohnishi ${ }^{10}$, S. Okukawa ${ }^{5}$, F. Orozco ${ }^{4}$, A. Oshima ${ }^{11}$, M. Raljevich ${ }^{3}$, H. Rivera ${ }^{3}$, T. Saito ${ }^{18}$, Y. Sakakibara ${ }^{5}$, T. Sako $^{10}$, T. K. Sako ${ }^{10}$, S. Shibata ${ }^{12}$, A. Shiomi ${ }^{16}$, M. Subieta ${ }^{3}$, N. Tajima ${ }^{19}$, W. Takano ${ }^{6}$, M. Takita ${ }^{10}$, Y. Tameda ${ }^{20}$, K. Tanaka ${ }^{21}$, R. Ticona $^{3}$,

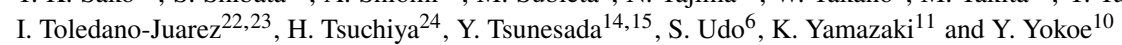

${ }^{1}$ Departamento de Física, CUCEI, Universidad de Guadalajara, Guadalajara, Jalisco, Mexico. ${ }^{2}$ Department of Physics, Shinshu University, Matsumoto, Nagano 390-8621, Japan. ${ }^{3}$ Instituto de Investigaciones Físicas, Universidad Mayor de San Andrés, La Paz 8635, Bolivia. ${ }^{4}$ Doctorado en Tecnologías de la Información, CUCEA, Universidad de Guadalajara, Zapopan, Jalisco, Mexico. ${ }^{5}$ Graduate School of Engineering Science, Yokohama National University, Yokohama, Kanagawa 240-8501, Japan. ${ }^{6}$ Faculty of Engineering, Kanagawa University, Yokohama, Kanagawa 221-8686, Japan. ${ }^{7}$ Utsunomiya University, Utsunomiya, Tochigi 3218505, Japan. ${ }^{8}$ Departamento de Tecnologíias de la Información, CUCEA, Universidad de Guadalajara, Zapopan, Jalisco, Mexico. ${ }^{9}$ Faculty of Engineering, Yokohama National University, Yokohama, Kanagawa 240-8501, Japan. ${ }^{10}$ Institute for Cosmic Ray Research, University of Tokyo, Kashiwa, Chiba 277-8582, Japan. ${ }^{11}$ College of Engineering, Chubu University, Kasugai, Aichi 487-8501, Japan. ${ }^{12}$ Chubu Innovative Astronomical Observatory, Chubu University, Kasugai, Aichi 487-8501, Japan. ${ }^{13}$ Departamento de Ciencias de la Información y Desarrollo Tecnológico, Cutonala, Universidad de Guadalajara, Tonalá, Jalisco, Mexico. ${ }^{14}$ Graduate School of Science, Osaka City University, Osaka, Osaka 558-8585, Japan. ${ }^{15} \mathrm{Nambu}$ Yoichiro Institute for Theoretical and Experimental Physics, Osaka City University, Osaka, Osaka 558-8585, Japan. ${ }^{16}$ College of Industrial Technology, Nihon University, Narashino, Chiba 275-8576, Japan. ${ }^{17}$ National Institute of Informatics, Chiyoda, Tokyo 101-8430, Japan. ${ }^{18}$ Tokyo Metropolitan College of Industrial Technology, Arakawa, Tokyo 116-8523, Japan. ${ }^{19}$ RIKEN, Wako, Saitama 351-0198, Japan. ${ }^{20}$ Faculty of Engineering, Osaka ElectroCommunication University, Neyagawa, Osaka 572-8530, Japan. ${ }^{21}$ Graduate School of Information Sciences, Hiroshima City University, Hiroshima, Hiroshima 731-3194, Japan. ${ }^{22}$ Doctorado en Ciencias Físicas, CUCEI, Universidad de Guadalajara, Guadalajara, Jalisco, Mexico. ${ }^{23}$ Maestría en Ciencia de Datos, Departamento de Métodos Cuantitativos, CUCEA, Universidad de Guadalajara, Zapopan, Jalisco, Mexico. ${ }^{24}$ Japan Atomic Energy Agency, Tokai-mura, Ibaraki 319-1195, Japan. 